\title{
Diagnostic schemes for reducing epidemic size of african viral hemorrhagic fever outbreaks
}

\author{
Iruka N Okeke ${ }^{1}$, Robert S Manning ${ }^{2}$, Thomas Pfeiffer ${ }^{3}$ \\ ${ }^{1}$ Department of Biology, Haverford College, Haverford, PA, United States \\ ${ }^{2}$ Department of Mathematics, Haverford College, Haverford, PA, United States \\ ${ }^{3}$ NZ Institute for Advanced Study, Massey University, Auckland, New Zealand
}

\begin{abstract}
Introduction: Viral hemorrhagic fever (VHF) outbreaks, with high mortality rates, have often been amplified in African health institutions due to person-to-person transmission via infected body fluids. By collating and analyzing epidemiological data from documented outbreaks, we observed that diagnostic delay contributes to epidemic size for Ebola and Marburg hemorrhagic fever outbreaks.

Methodology: We used a susceptible-exposed-infectious-removed (SEIR) model and data from the 1995 outbreak in Kikwit, Democratic Republic of Congo, to simulate Ebola hemorrhagic fever epidemics. Our model allows us to describe the dynamics for hospital staff separately from that for the general population, and to implement health worker-specific interventions.

Results: The model illustrates that implementing World Health Organization/US Centers for Disease Control and Prevention guidelines of isolating patients who do not respond to antimalarial and antibacterial chemotherapy reduces total outbreak size, from a median of 236, by $90 \%$ or more. Routinely employing diagnostic testing in post-mortems of patients that died of refractory fevers reduces the median outbreak size by a further $60 \%$. Even greater reductions in outbreak size were seen when all febrile patients were tested for endemic infections or when febrile health-care workers were tested. The effect of testing strategies was not impaired by the 1-3 day delay that would occur if testing were performed by a reference laboratory.

Conclusion: In addition to improving the quality of care for common causes of febrile infections, increased and strategic use of laboratory diagnostics for fever could reduce the chance of hospital amplification of VHFs in resource-limited African health systems.
\end{abstract}

Key words: modeling; viral hemorrhagic fever; Ebola hemorrhagic fever; outbreak; health workers.

J Infect Dev Ctries 2014; 8(9):1148-1159. doi:10.3855/jidc.4636

(Received 30 December 2013 - Accepted 11 March 2014)

Copyright (C) 2014 Okeke et al. This is an open-access article distributed under the Creative Commons Attribution License, which permits unrestricted use, distribution, and reproduction in any medium, provided the original work is properly cited.

\section{Introduction}

Febrile illnesses or fevers are often the result of viral, bacterial or parasitic infection. It is impossible to determine the precise etiology of many febrile illnesses based on signs and symptoms alone. Diagnosis can usually only be confirmed with laboratory support, even after thorough clinical evaluation and history-taking, and with knowledge of local endemic pathogens. In many parts of Africa febrile illnesses are common but treatment is almost always prescribed after syndromic diagnoses. Fevers are invariably diagnosed as malaria at first presentation in malaria-endemic areas, where an alternative diagnosis is often proposed only when firstor second-line antimalarial chemotherapy fails. Diagnosis by sequential treatment leads to overuse of valuable antimicrobials and is detrimental to the management of infections caused by bacteria and by other causes of fever [1-5]. Despite these shortcomings, and because laboratory diagnostics are often unavailable, diagnosis by sequential treatment is also recommended for life-threatening, blood-borne viral hemorrhagic fevers (VHFs) [6], such as the illnesses caused by filoviruses Ebola and Marburg as well as Lassa and Lujo arenaviruses, where it contributes to diagnostic delay.

Hemorrhagic viruses are devastating but relatively uncommon causes of fever in Africa and are therefore not routinely considered in primary differential diagnoses unless an outbreak is known to be in progress. Because person-to-person transmission via infected body fluids is common, delays to detection mean that hospitals can and do amplify epidemics when they occur [7,8]. Routine hospital infection control precautions can prevent the spread of most pathogens within hospitals but for some high-risk agents, special barrier precautions, such as isolation of patients and post-contact decontamination of health 
workers, are necessary. These procedures are routinely used when patients in affluent countries fall ill following a recent visit to malaria and hemorrhagic virus endemic areas, accounting for why numerous exported VHF patients have not initiated outbreaks abroad [9-11].

Most hospitals in equatorial Africa do not routinely isolate febrile patients nor do they routinely use strict barrier precautions. While hospital-amplified VHF outbreaks have been linked to unacceptable needle-sharing and absence of basic infection control precautions in some institutions [7,12,13], the vast majority of documented VHF outbreaks involving hospitals have seen nosocomial transmission due to inadequate isolation facilities and because gloves and expensive protective clothing were not always used by attending personnel [13-16]. As most in-patients in often overcrowded, under-resourced and understaffed hospitals in equatorial Africa have a fever of infectious origin, highest-level barrier precautions cannot feasibly be implemented, nor do most common infections require them. Notwithstanding, appropriate barrier practices can be, and have been, rapidly instituted in African hospitals during hemorrhagic fever outbreaks [16-19], and have led to rapid abatement of all documented Ebola and Marburg outbreaks $[20,21]$.

In such a setting, early detection of VHFs can reduce the size of hospital-amplified outbreaks considerably. This is illustrated by a 2004 outbreak in Yambio, Sudan, where health workers noticed a cluster of cases and suspected that a filovirus endemic might have begun [22]. In an exemplary case of deductive epidemiology combined with access to testing facilities in nearby Kenya the epidemic was identified very rapidly. As shown in Table 1, the reported case number from Yambio was much smaller than most outbreaks that saw diagnostic delays of longer than a month. Smaller outbreaks have also included fewer infected health workers (Table 1).

Post-outbreak assessment of a 2000 Uganda outbreak in Gulu, in which 425 people contracted Ebola virus, proposed that an "astute health worker", who could recognize anomalies in fever patients might be the key to early detection [23]. However VHFs often do not present symptoms different from those seen in malaria, typhoid fever and other infections at early stages and the clinical case definitions for Ebola and Marburg hemorrhagic fevers are unreliable $[8,18,23,24]$. The Uganda outbreak eventually came to light because physicians did detect an anomaly, but that was a spike in the number of deaths, by which time the epidemic was well underway [14,25]. Recognizing unusual cases early, without epidemiological context, in an outbreak is difficult for even the most skilled diagnostician when such cases

Table 1: Outbreak size, fatalities, time to detection and numbers of health workers infected in documented filovirus outbreaks without clear evidence of needle-reuse. It is probable that only a fraction of outbreaks are detected and reported even though VHFs are classified as notifiable diseases [49]. An outbreak at the Masango Hospital, for instance, was only documented retrospectively, following investigation of the widely publicized 1995 connected outbreak in Kikwit [25] and a Lassa fever outbreak in Nigeria was only confirmed after death of the last infected case, a physician [7]. Moreover, just as outbreaks may be unreported, case reporting within recorded outbreaks is likely underestimated.

\begin{tabular}{|c|c|c|c|c|}
\hline Outbreak (Year: Viral Strain, location) & Cases & $\begin{array}{l}\text { Fatalities (Case } \\
\text { fatality } \% \text { ) }\end{array}$ & $\begin{array}{c}\text { Proportion of } \\
\text { infected that were } \\
\text { health workers }\end{array}$ & $\begin{array}{l}\text { Time to } \\
\text { detection }\end{array}$ \\
\hline $\begin{array}{l}\text { 1995: Ebola Zaire in Kikwit, Democratic Republic of Congo } \\
{[29,50]}\end{array}$ & 315 & $247(78.4)$ & $25.4 \%(80 / 315)$ & 4.5 months \\
\hline $\begin{array}{l}\text { 1998: Marburg in Durba, Watsa Zone, Democratic republic } \\
\text { of the Congo [51] }\end{array}$ & 154 & $125(81.2)$ & $0.6 \%(7 / 154)$ & 6 months $*, * *$ \\
\hline 2000: Ebola Sudan in Gulu, Uganda $[14,23,52]$ & 425 & $224(52.7)$ & $7.3 \%(31 / 425)$ & 1.5 months \\
\hline $\begin{array}{l}\text { 2003: Ebola in Kéllé district, Democratic republic of the } \\
\text { Congo [33] }\end{array}$ & 143 & $128(89.5)$ & Unknown & 30 days \\
\hline 2004: Ebola in Yambio, Sudan [22,34] & 17 & $7(41.2)$ & $11.8 \%(2 / 17)$ & 10 days \\
\hline 2005: Marburg in Uige, Angola [15] & 374 & $158(42.2)$ & $4.8 \%(18 / 374)$ & $\geq 1$ month \\
\hline $\begin{array}{l}\text { 2005: Ebola Zaire in Etoumbi district Democratic republic of } \\
\text { the Congo [55] }\end{array}$ & 12 & $10(83.3)$ & 0 & 1 month \\
\hline
\end{tabular}

*Civil conflict restricted access to the outbreak location

**Multiple simultaneous outbreaks confirmed 
present amidst similar 'detractor' cases [26].

Most of the available literature relating to preventing VHF outbreaks focuses on containing outbreaks that have already been initiated. We used a mathematical model to address interventions that could be implemented before an outbreak occurred and lead to early detection of VHF outbreaks, with the added benefit of increasing the overall quality of care in resource-limited hospitals.

Laboratory diagnostic options for early detection of Ebola and other hemorrhagic viruses exist but have limitations. Gold standard viral amplification can only be performed in biosafety level-4 laboratories but antibody and nucleic-acid based tests that can use inactivated specimens can be used in the field and at district hospitals $[18,19,27]$. Nucleic-acid based tests, such as RT-PCR offer the most promise for use in African hospitals but require molecular biology facilities that are far from ubiquitous.

Although costs for such tests are falling, they remain too high for resource limited clinics to use routinely for every febrile diagnosis today. One way to identify epidemics early might therefore be to identify informative patient sub-populations that could be screened routinely. Because data from earlier outbreaks suggests that health workers likely represent such a sub-population, we here extend a previous SEIR model for Ebola outbreaks [28] to model disease dynamics in health workers separately from the general population. We then use the model to simulate outbreaks and their detection via different diagnostic protocols.

\section{Methodology}

\section{The modified SEIR model}

Ebola outbreaks have previously been modeled using a basic susceptible-exposed-infectious-removed (SEIR) design [28]. In such a model, the population is described by four compartments which contain susceptible, exposed, infectious and removed individuals (Figure 1). The dynamics of an epidemic are determined by three processes: 1. Susceptible individuals may get exposed through contact with infectious ones. The rate of this process is assumed to be $b I / N$, where $I$ is the number of infectious individuals, $\mathrm{N}$ is the total population size, and $\mathrm{b}$ is the rate constant associated with infection.

2. Exposed individuals are those individuals that are infected but have not yet developed symptoms and are therefore not yet infectious. As the disease progresses, exposed individuals become infectious. This process is described by the rate $\mathrm{k}$.
Figure 1. Schematic representation of the basic SEIR model. The model has four compartments, representing susceptible (S), exposed (E), infectious (I) and recovered/removed (R) individuals.

\section{SEIR model}
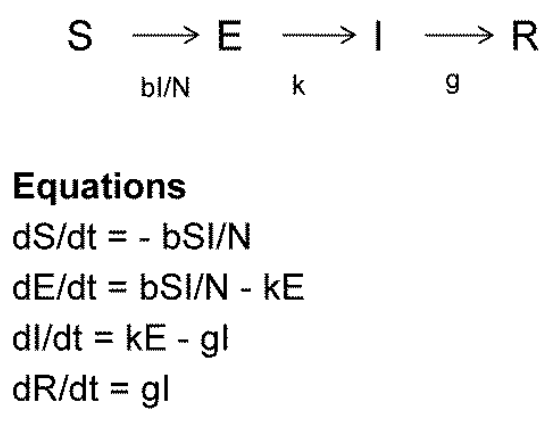

\section{Parameters}

$b$ - transmission in general population

$\mathrm{k}$ - disease progression

$\mathrm{g}-$ recovery/death

Figure 2. Schematic representation of the modified SEIR model developed for hypothesis testing in this study. Our model extends a simple SEIR model to two subpopulations, the general population and health workers. While disease-specific parameters are the same for both subpopulations, they are assumed to differ in their exposure, resulting in different infection rates, $b_{1}$ and $b_{2}$.

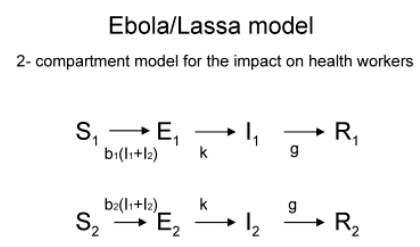

Equations

$\mathrm{dE}_{1} / \mathrm{dt}=\mathrm{b}_{1}\left(\mathrm{I}_{1}+\mathrm{I}_{2}\right)-\mathrm{kE}_{1}$ $\mathrm{dl}_{1} / \mathrm{dt}=\mathrm{kE}_{1}-\mathrm{gl}_{1}$ $\mathrm{dE}_{2} / \mathrm{dt}=\mathrm{b}_{2}\left(\mathrm{I}_{1}+\mathrm{I}_{2}\right)-\mathrm{kE}$ $\mathrm{dl}_{2} / \mathrm{dt}=\mathrm{kE}_{2}-\mathrm{gl}_{2}$

Relation to Chowell et al, 2004 $\mathrm{dE} / \mathrm{dt}=\mathrm{d}\left(\mathrm{E}_{1}+\mathrm{E}_{2}\right) / \mathrm{dt}=\left(\mathrm{b}_{1}+\mathrm{b}_{2}\right) \mathrm{I}-\mathrm{kE}$ $\mathrm{dl} / \mathrm{dt}=\mathrm{d}\left(\mathrm{I}_{1}+\mathrm{I}_{2}\right) / \mathrm{dt}=\mathrm{kE}-\mathrm{gl}$

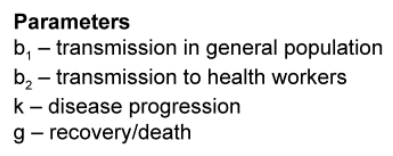

Parameter values from Chowell et al, 2004 $b=b_{1}+b_{2}=0.33$ (per day, pre-intervention) $b=b_{1}+b_{2}=0.09$ (per day, post-intervention) $k=0.19$ (per day) $\mathrm{g}=0.18$ (per day)

3. Infectious individuals in turn are assumed to progress into removed individuals at rate $g$, either through recovery or death (Figure 1). For Ebola outbreaks, a SEIR design has been used to estimate epidemic parameters [28]. More detailed approaches include a six-compartment model, which allows for a separate compartment of hospitalized cases as well as a category of infectious cadavers [20]. 
To separately model individuals within health centers, such as health workers, who likely play a significant role in the disease dynamics, we here extend the basic SEIR model to two sub-populations: the general population and health workers. Within each of these groups, there are four subtypes: susceptible, exposed, infectious and removed. The resulting eight categories are denoted by $\mathrm{S}_{1}, \mathrm{E}_{1}, \mathrm{I}_{1}, \mathrm{R}_{1}$, $S_{2}, E_{2}, I_{2}$ and $R_{2}$, where index 1 stands for the general population and index 2 for the health worker population. A schematic representation of the model is show in Figure 2.

The rates $\mathrm{k}$ and $\mathrm{g}$ are assumed to be the same for health workers and the general population. This is because they are mainly determined by diseasespecific factors. In principle these rates might differ between hospitalized and non-hospitalized population, for instance because hospitalized patients might have weakened immunity, which in turn could affect the dynamics of hospital amplification; this cannot be captured by our approach. The two populations are assumed to be similar in their infectiousness, i.e. infectious members of both populations are equally likely to transmit the disease to others. However, health workers are assumed to differ from the general population in their exposure, leading to different transmission rates $b_{1}$ and $b_{2}$ in the two subpopulations.

For outbreaks that affect only a small proportion of the population, it can be assumed that the vast majority of the population remain in the pool of susceptible individuals, i.e. $\mathrm{S} \approx \mathrm{N}$. This has been shown to apply to the 1995 outbreak in Kikwit [28] and allows to simplify the model to the following system of four linear ordinary differential equations:

$$
\begin{aligned}
& \mathrm{dE}_{1} / \mathrm{dt}=\mathrm{b}_{1}\left(\mathrm{I}_{1}+\mathrm{I}_{2}\right)-\mathrm{kE}_{1} \\
& \mathrm{dI}_{1} / \mathrm{dt}=\mathrm{kE}_{1}-\mathrm{gI}_{1} \\
& \mathrm{dE}_{2} / \mathrm{dt}=\mathrm{b}_{2}\left(\mathrm{I}_{1}+\mathrm{I}_{2}\right)-\mathrm{kE}_{2} \\
& \mathrm{dI}_{2} / \mathrm{dt}=\mathrm{kE}_{2}-\mathrm{gI}_{2}
\end{aligned}
$$

\section{Model parameters}

The dynamics for the entire population in this model is the same as in the SEIR model used by Chowell et al [28]:

$$
\begin{aligned}
& \mathrm{dE} / \mathrm{dt}=\mathrm{d}\left(\mathrm{E}_{1}+\mathrm{E}_{2}\right) / \mathrm{dt}=\left(\mathrm{b}_{1}+\mathrm{b}_{2}\right) \mathrm{I}-\mathrm{kE} \\
& \mathrm{dI} / \mathrm{dt}=\mathrm{d}\left(\mathrm{I}_{1}+\mathrm{I}_{2}\right) / \mathrm{dt}=\mathrm{kE}-\mathrm{gI}
\end{aligned}
$$

For the total population, our model and the model by Chowell et al. [28] therefore result in exactly the same predictions. However, in contrast to the model by Chowell et al. [28], our model explicitly describes the dynamics in the health worker subpopulation. This extension requires an additional parameter describing
Figure 3. Cumulative cases over time for the 1995 outbreak in Congo for the time span from March 1 to June 311995 $[28,29]$. Black symbols stand for total cases, gray symbols for health workers. The lines show a linear regression of the logtransformed cumulative cases vs. time until interventions were implemented on May 12 [29]. The outbreak in this period of time shows approximately exponential growth. The growth rate is approximately the same in both subpopulations. Approximately $39 \%$ of the cases during this period were health workers.

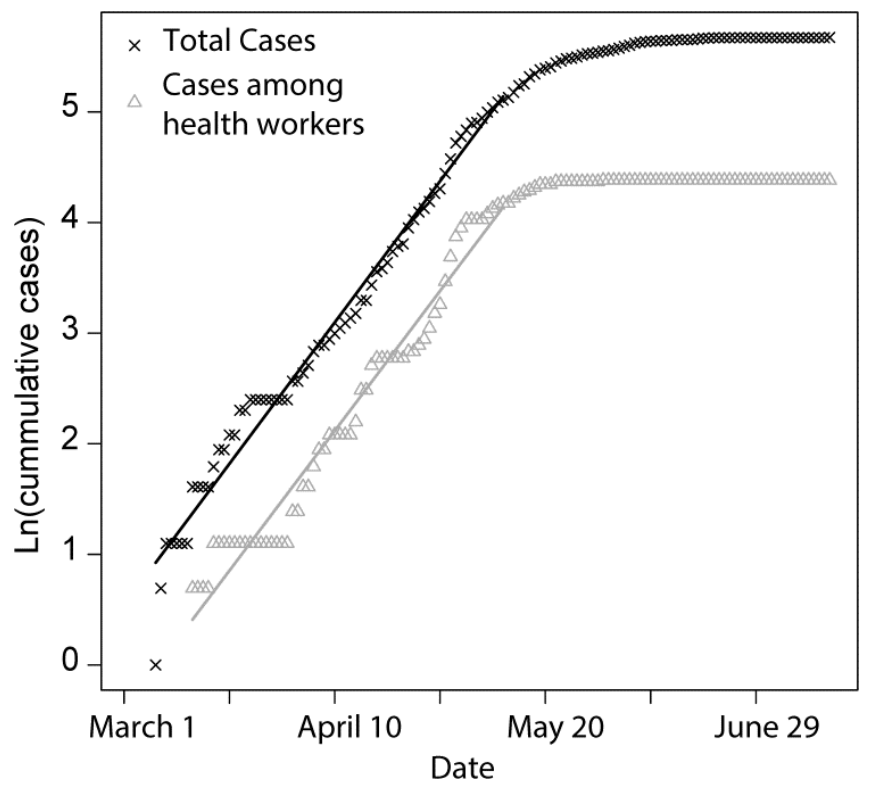

how the different subpopulations differ in their exposure, and allows us to simulate diagnostic schemes that differentiate between the two subpopulations.

Because both models result in the same overall dynamics, we apply the parameter values as obtained by Chowell et al. [28] to our model: disease progression rate $\mathrm{k}=0.19\left(\right.$ day $\left.^{-1}\right)$; recovery/death rate $\mathrm{g}$ $=0.18\left(\right.$ day $\left.^{-1}\right)$; pre-intervention infection rate $b=b_{1}+b_{2}$ $=0.33\left(\right.$ day $\left.^{-1}\right)$; and post-intervention infection rate $\mathrm{b}=$ $b_{1}+b_{2}=0.09\left(\right.$ day $\left.^{-1}\right)$. These estimates are based on data from the nosocomial phase of the 1995 Kikwit outbreak.

To calculate the relative contributions of the two subpopulations $b_{1}$ and $b_{2}$ to the overall infection rate $b$, we use in the following the health-worker specific data as given in Chowell et al [28]. The linear dynamics used in our model implies that before interventions are implemented, the outbreak grows exponentially in both sub-populations at the same rate. This is in good agreement with the observed dynamics: the growth rate of cumulative cases among the health workers is approximately the same as the growth rate in the entire population (see Figure 3). Since the number of new infections among the health workers is a constant 
fraction $b_{2} /\left(b_{1}+b_{2}\right)$ of the overall new infections and the outbreak started from a near disease-free population, the ratio between transmission rate to health workers $b_{2}$ and the overall transmission rate $b_{1}+b_{2}$ is the same as the ratio of cumulative health worker cases to total cumulative cases until interventions. This ratio is approximately 0.39 , which gives pre-intervention transmission rates of $b_{1}=0.2$ $\left(\right.$ day $\left.^{-1}\right)$ and $b_{2}=0.13\left(\right.$ day $\left.^{-1}\right)$. Note that if the health worker population was protected from infection, the value for $\mathrm{R}_{0}=\mathrm{b}_{1} / \mathrm{g}$ would be close to 1 , rather than 1.8 as estimated by Chowell et al [28] for the population with unprotected health workers. This highlights the role health worker-infections play for the disease dynamics.

After interventions were implemented, a total of 129 new cases were observed, 16 of which were health workers $[28,29]$. These new cases include individuals who were exposed before and after the implementation of interventions. At the time when interventions where implemented, the number of new cases was about 8 per day $[28,29]$. Given that the number of new cases equals $\mathrm{kE}$, one would expect at this point about 40 exposed individuals, 16 of which are health workers.
Thus while a substantial number of individuals from the general population became exposed postintervention, likely no or very few health workers were infected. This suggests a high success of health worker precautions for this particular epidemic, and indeed the WHO reported no new health worker cases among those who used post-intervention barrier nursing practices in that epidemic, with one case due to an inappropriate use of those practices [6]. Given a post-intervention transmission rate of $b=0.09\left(\right.$ day $\left.^{-1}\right)$ as estimated by Chowell et al. [28], we therefore use post-intervention values of $b_{1}=0.09\left(\right.$ day $\left.^{-1}\right)$ and $b_{2}=0$, respectively.

\section{Stochastic simulations}

We use the above parameters to perform stochastic simulations of outbreaks for different diagnostic protocols. We start the simulations with a single exposed individual in the general population. For each time step (day) we determine for both streams the number of susceptible individuals becoming exposed, exposed becoming infectious, and infectious being removed (recovered or deceased). The numbers of individuals becoming exposed, $\mathrm{B}_{1}(\mathrm{t})$ and $\mathrm{B}_{2}(\mathrm{t})$ are

Table 2: Diagnostic scenarios for VHF tested using the SEIR model with two sub-populations. Baseline diagnostic probabilities $\left(\mathrm{p}_{1}\right.$ and $\mathrm{p}_{2}$ ) assume an "astute observer" calibrated from the Kikwit outbreak (see text). For other scenarios, we assume an $80 \%$ hospitalization rate for the general population and $100 \%$ for health workers, compounded by other effects in some scenarios: a $25 \%$ chance of an infected surviving the 15 -day delay in CDC and a $80 \%$ mortality rate in PMT. Included delays $\left(\mathrm{d}_{1}\right.$ and $\left.\mathrm{d}_{2}\right)$ assume that malaria is diagnosed by point-of-care rapid diagnostic test or microscopy (return results on the same day), bacteria are detected by culture (returning results in 2 days) and hemorrhagic viruses are detected by RT-PCR at a reference laboratory (1 day).

\begin{tabular}{lcccc}
\hline Scenario & Refs & $\mathbf{p}_{\mathbf{1}}$ & $\mathbf{p}_{\mathbf{2}}$ & $\mathbf{d}_{\mathbf{1}}$ \\
\hline Baseline: As unfolded in Kikwit, 1995 (Note 1) & {$[29]$} & 0.006 & 0.006 & 0 \\
WHO/ CDC (Note 2) & {$[6]$} & 0.2 & 0.25 & 15 \\
Utopian scenario (UTS; Note 3) & {$[9,11,57]$} & 0.8 & 1 & 1 \\
Febrile Health Worker (FHS; Note 4) & This study & 0.006 & 1 & 1 \\
Post-mortem testing (PMT; Note 5) & This study & 0.64 & 0.8 & 9 \\
Routine testing (RT; Note 6): & This study & 0.8 & 1 & 9 \\
\hline
\end{tabular}

Note 1: Baseline diagnostic probabilities (p1 and p2) assume an "astute observer" calibrated from the Kikwit outbreak (see text). For other scenarios, we assume an $80 \%$ hospitalization rate for the general population and $100 \%$ for health workers, compounded by other effects in some scenarios: a $25 \%$ chance of an infected surviving the 15-day delay in CDC and a $80 \%$ mortality rate in PMT. Included delays (d1 and d2) assume that malaria is diagnosed by point-ofcare rapid diagnostic test or microscopy (return results on the same day), bacteria are detected by culture (returning results in 2 days) and hemorrhagic viruses are detected by RT-PCR at a reference laboratory (1 day).

Note 2: $\mathrm{WHO} / \mathrm{CDC}$ recommendations for infection control in the African health care setting (CDC), recommending treatment failure for malaria and systemic bacterial infection as the means for identifying presumptive VHF cases. We conservatively selected two weeks for serial chemotherapy for malaria and bacterial infections. In areas where resistance is common, serial chemotherapy for refractory infections can continue for much longer periods.

Note 3: Everyone who becomes febrile within an endemic/high risk area is isolated immediately and tested or treated until diagnosis or discharge. This 'Utopian' protocol is currently employed in affluent countries for patients recently returning from Africa. Isolation of all febrile patients in an endemic area, even for a short duration, would be infeasible.

Note 4: Health workers are tested for a range of systemic pathogens, including endemic hemorrhagic viruses, whenever they are febrile. No diagnosis beyond baseline applied to non-health workers.

Note 5: Every febrile case that is managed according to the WHO/CDC recommended protocol described above but dies is tested post-mortem. We allowed one week to death of the first such case in the model.

Note 6: The hospital uses routine laboratory tests for highly prevalent endemic infection (malaria and bacteria). Patients who test negative are isolated immediately and then tested for VHF. 
drawn from a Poisson distribution with a mean of $\mathrm{b}_{1}\left(\mathrm{I}_{1}(\mathrm{t})+\mathrm{I}_{2}(\mathrm{t})\right)$ and $\mathrm{b}_{2}\left(\mathrm{I}_{1}(\mathrm{t})+\mathrm{I}_{2}(\mathrm{t})\right)$. The numbers of exposed becoming infectious, $\mathrm{K}_{1}(\mathrm{t})$ and $\mathrm{K}_{2}(\mathrm{t})$, are drawn from a binomial distribution with a probability parameter of $\mathrm{k}$, and a number of $\mathrm{E}_{1}(\mathrm{t})$ and $\mathrm{E}_{2}(\mathrm{t})$ trials. The numbers of infectious becoming recovered/removed, $\mathrm{G}_{1}(\mathrm{t})$ and $\mathrm{G}_{2}(\mathrm{t})$, are drawn from a binomial distribution with a probability parameter of $\mathrm{g}$, and a number of $\mathrm{I}_{1}(\mathrm{t})$ and $\mathrm{I}_{2}(\mathrm{t})$ trials. The overall dynamics is updated as

$$
\begin{aligned}
& E_{1}(t+1)=E_{1}(t)+B_{1}(t)-K_{1}(t) \\
& I_{1}(t+1)=I_{1}(t)+K_{1}(t)-G_{1}(t) \\
& E_{2}(t+1)=E_{2}(t)+B_{2}(t)-K_{2}(t) \\
& I_{2}(t+1)=I_{2}(t)+K_{2}(t)-G_{2}(t)
\end{aligned}
$$

\section{Outbreak detection}

Depending on whether they are members of the general population or health workers, each individual that becomes infectious is assumed to be correctly diagnosed at a probability of $\mathrm{p}_{1}$ and $\mathrm{p}_{2}$, respectively. If an individual is diagnosed correctly, it will take a time of $\mathrm{d}_{1}$ and $\mathrm{d}_{2}$, respectively, from the day that individual becomes infectious until interventions are implemented and the outbreak dynamics change from pre-intervention to post-intervention. The values for these probabilities and delays depend on the diagnostic protocol and are given in Table 2. Once a VHF has been diagnosed, we assume that interventions are implemented without further delay.

As reference scenario we use a simple "astute observer" [14] model. At a certain probability $\mathrm{p}_{1}$ a given general-population case will lead an observer, that is an astute health worker, to conclude that an Ebola epidemic is in process, and at a potentially different probability $\mathrm{p}_{2}$ a given health worker case will lead to the same conclusion. In the case of the 1995 Kikwit epidemic, we assume that no special attention was paid to health workers (as seems to have been the case), and thus set $\mathrm{p}_{1}=\mathrm{p}_{2}$. The expected number of total cases until detection in this case is $1 / p_{1}$. With a total of 154 cases at time of intervention, this means that $\mathrm{p}_{1}=0.006$. Certainly this is a rough model for detection, but we note that our main concern is comparing various more sensitive and specific detection strategies against this baseline, so that using a different model for the baseline should not have a major effect on our conclusions about the relative effects of other detection strategies on the size of the outbreak.

In addition to the reference scenario, we used our model to evaluate the diagnostic scenarios shown in
Table 2, and compared the outbreak size from these interventions to that obtained from the reference scenario on the Kikwit outbreak. In each case, once a VHF outbreak has been detected, we switch from preintervention to post-intervention parameters. The latter model isolation and barrier nursing of all cases and probable cases along the lines specified in the $\mathrm{WHO} /$ US Centers for Disease Control and Prevention (CDC) guidelines for African settings [6].

The diagnostic scenarios shown in Table 2 differ in the probabilities $p_{1}$ and $p_{2}$ at which a case in the general population and the health worker subpopulation is detected and in the time $d_{1}$ and $d_{2}$ it takes from onset of symptoms to diagnosis. As the detection probability, the delay depends on the subpopulation, and is denoted by $\mathrm{d}_{1}$ and $\mathrm{d}_{2}$.

In summary, we use 6 different diagnostic scenarios. In the baseline scenario, are described above, we use a detection probability of $\mathrm{p}_{1}=\mathrm{p}_{2}=0.006$, and do not explicitly model a delay (i.e. $\mathrm{d}_{1}=\mathrm{d}_{2}=0$ ). In the $\mathrm{WHO} / \mathrm{CDC}[\mathrm{CDC}]$ scenario, we assume a long delay of $d_{1}=d_{2}=15$ because serial chemotherapy for malaria and bacterial infections needs to fail before VHF's are considered. We assume that after such a delay, only $20 \%$ of cases from the general population and $25 \%$ of the health worker cases are available for further diagnosis. (The difference in $\mathrm{p}_{1}=0.2$ and $\mathrm{p}_{2}=0.25$ reflects the assumption that about $80 \%$ of the cases from the general population but $100 \%$ of the health worker cases are hospitalized at some point during sickness). In the 'utopian' scenario (UTS), we assume high detection probabilities of $\mathrm{p}_{1}=0.8$ and $\mathrm{p}_{2}=1.0$ and a diagnostic delay of only one day. In a scenario that specifically targets infectious health workers (FHS), we use baseline assumptions for the general population but UTS assumptions for health workers. Moreover, one scenario investigates postmortem diagnosis (PMT), where we assume a relatively long delay of 9 days, and intermediate detection probabilities of $\mathrm{p}_{1}=0.64$ and $\mathrm{p}_{2}=0.8$; and one scenario investigates routine testing of all patients that are tested negative for malaria and bacteria, implying a delay of 3 days and detection probabilities of $\mathrm{p}_{1}=0.8$ and $\mathrm{p}_{2}=1.0$.

\section{Ethics statement}

This study was not submitted to or reviewed by our institutional review boards because it does not involve research using humans or human specimens. 


\section{Results}

\section{Comparison of diagnostic interventions}

Figure 4 demonstrates that in an outbreak modeled after the one in Kikwit, adhering to the WHO/CDC [CDC] recommendation [6] of isolating patients who do not recover after a single round of chemotherapy for malaria and systemic bacterial infection can reduce outbreak size from a baseline median of 236 to a median of 24, using a conservative intervention that assumes a best case scenario in which the necessary drugs are available and quality-assured and that acquired antimicrobial resistance does not exist, and modeling the waiting time for the malaria and bacterial infection treatment as a 15-day delay before intervention. In most African hospitals medicine stock-outs and substandard drugs are occasional or even frequent occurrences. Additionally, febrile patients receive multiple chemotherapeutic courses because antimicrobial resistance is becoming more common and culture and susceptibility testing facilities are largely unavailable [8,30]. Recent information from African settings suggests that drug resistance in a febrile illness of bacterial origin can increase diagnostic delay to 30 days or longer [30]. Such extended delay in our model results in approximately a three-fold increase in VHF epidemic size if the basic WHO/CDC protocol is employed for detection and initiation of interventions (data not shown). On a positive note, routine rapid diagnostic malaria testing at all levels of the health system is currently being rolled out in multiple African countries and has been successfully implemented in Senegal [31]. We modeled a protocol that included routine rapid-diagnostic testing for malaria but no testing for any other febrile illnesses, reducing the delay to 8 days. We observed a $40 \%$ reduction in VHF epidemic size below that seen with a 15-day delay in the CDC simulation (data not shown).

When we used additional laboratory diagnostic testing to enhance VHF detection, we saw considerable reduction in outbreak size. As expected, the smallest outbreak sizes were obtained in the scenario where all hospitalized febrile patients are tested (UTS). This 'Utopian' model is used for febrile patients returning to Europe or North America from malaria/filovirus endemic areas and probably contributes to the fact that cases are exported but outbreaks are not. Because this protocol may be difficult to implement in remote district hospitals, where VHF outbreaks are most likely to occur, we evaluated scenarios in which non-molecular testing for more common confounding diagnoses was performed
Figure 4. Outbreak sizes for different diagnostic scenarios. For each parameter set, 10000 stochastic simulations were performed. The bars show the $10 \%-90 \%$ quantiles and the median outbreak sizes, the cross shows the mean outbreak sizes. Only detected outbreaks were included - for our model parameters, a substantial fraction of outbreaks die out before being detected. The base line detection probability is approximately 0.006 per case, and is assumed to be the same for the general population and health workers. This leads to outbreaks with a median size of about 230 cases. Adhering to the minimal $\mathrm{WHO} / \mathrm{CDC}$ recommendations for outbreak management in African settings can reduce outbreak sizes to a median of 24 cases (CDC). In an "utopian" scenario (UTS), where all febrile cases screened for VHF, and screening takes one day, (or, as more typically occurs in reality, are isolated until they are diagnosed or discharged) median outbreak size is reduced to a single case. Routine testing (RT), where febrile cases are initially tested for common parasitic and bacterial infections, and those patients with negative results are subsequently screened for VHF has compared to UTS a slightly increased median outbreak size of 3 cases, due to the additional delay from the initial test round. Outbreak sizes for febrile health worker testing (FHS), and post-mortem testing (PMT) fall between the outbreak sizes for the CDC scenario and RT scenario. The differences in outbreak sizes for the simulations shown here and in Fig 4 are statistically significant (t-tests on log-transformed outbreak sizes: $\mathrm{p}<0.001$ for all pairwise comparisons.)

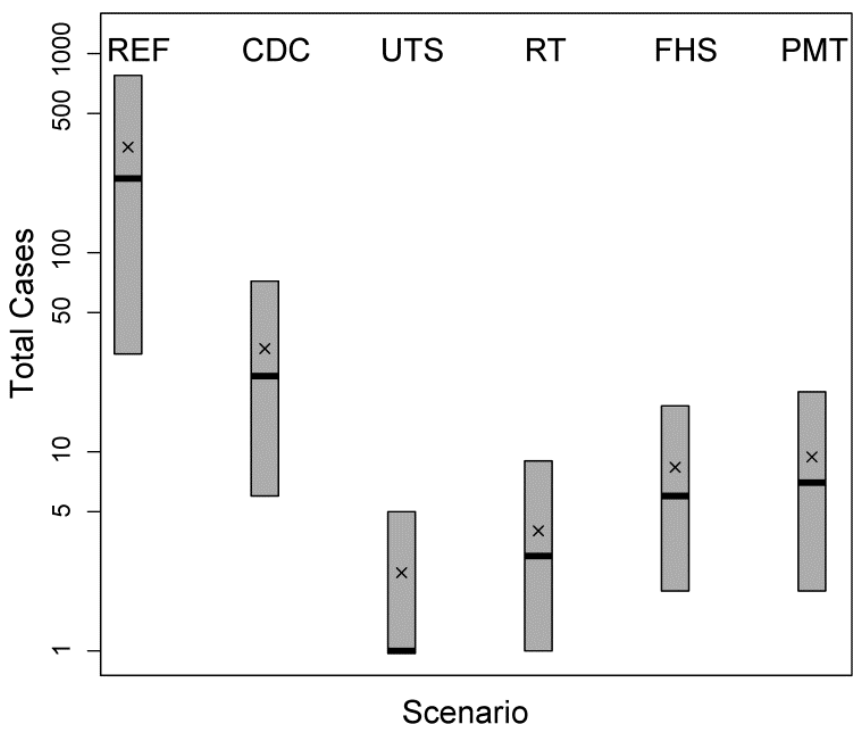

and/or only a subset of febrile patients were tested for VHFs.

Figure 4 demonstrates that testing all febrile patients for common parasitic and bacterial infections (such as rapid diagnostic tests for malaria and blood culture for bacteria for example), and implementing WHO/CDC recommended containment for patients who tested negative (RT) produced VHF outbreak sizes that were somewhat larger than, but comparable to, the 'Utopian' (UTS) scenario. This option would make it possible for resource-limited hospitals to 
enhance the management and surveillance of malaria and common bacterial infections whilst simultaneously reducing diagnostic delay for VHF outbreaks.

In the event that hospitals were unable to implement testing to this degree, we hypothesized that providing laboratory testing for health-workers who become febrile or post-mortem testing for febrile patients who did not recover in spite of chemotherapy would be another way to increase the chance that outbreaks are identified earlier and therefore are smaller. Health workers were selected as a potential sub-population for enhanced testing because their close contact with patients with VHF places them at increased risk for infection and opportunity to spread the disease within the hospital [8]. Mortality clusters have been instrumental for identifying outbreaks in the past $[8,14,29,32,33]$. Therefore we envisaged also that post-mortem identifications might occur more quickly if they were revealed by test results, before clustering became evident. As shown in Figure 4 both the febrile health worker testing [FHS] and post-mortem testing [PMT] interventions reduced outbreak size to levels below those seen with the minimal WHO/CDC [CDC] syndromic diagnosis recommendation, with slightly better results being seen with the FHS strategy.

Not every institution that would choose to implement testing strategies such as those that have been modeled would be equipped to perform qualityassured testing on-site. We therefore modeled the effect of additional delays that would result from using the same strategy with off-site testing at, for example, a regional laboratory serving multiple district health centers. Given that improved communications across Africa mean that results can be returned rapidly, if samples are routinely and regularly transported from the district health center to the testing facility, the delay afforded would be slight. We allowed one day for febrile health worker testing and 3 days for postmortems, consistent with the time-to-detection from local off-site labs in actual Ebola outbreaks $[22,33,34]$. These delays resulted in only a small increase in outbreak size (Figure 5). Therefore, in the event that on-site testing is not possible, off-site testing should be considered.

\section{Impact of detection probability and diagnostic delay on simulation results}

Our simulation results depend on a number of parameters. While for those parameters that describe the dynamics of an outbreak ( $b_{1}, b_{2}, k$ and g), we rely on estimates from previous outbreaks, the parameters
Figure 5. Impact of off-site vs. on-site testing. The scenarios are relatively robust to additional delays from off-site rather than on-site testing (one day for FHS and three days for PMT). Outbreak sizes increase only slightly, and are still below the outbreak sizes for the CDC scenario.

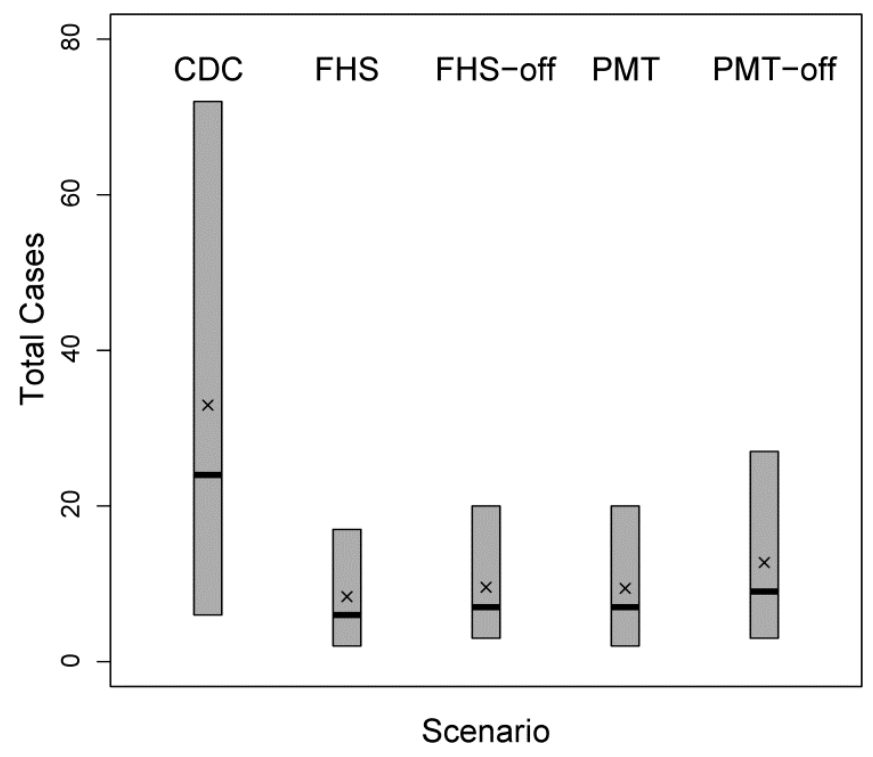

that characterize the diagnostic schemes $\left(\mathrm{d}_{1}, \mathrm{~d}_{2}, \mathrm{p}_{1}\right.$ and $\mathrm{p}_{2}$,) are chosen ad hoc. It is therefore of importance to analyze in more detail how these parameters determine outbreak sizes.

For those scenarios that do not differentiate, in terms of detection probability, between the two subpopulations (Ii.e $\mathrm{p}_{1} \approx \mathrm{p}_{2}=\mathrm{p}$ and $\mathrm{d}_{1} \approx \mathrm{d}_{2}=\mathrm{d}$ ), the expected outbreak size at the point of time when the individual that is eventually diagnosed correctly enters the infectious compartment is given by $1 / \mathrm{p}$. This means, for example, that in those scenarios where nearly every febrile case is tested for VHF's, the first infectious case will be detected. If $80 \%$ of potential cases are not evaluated, on average the fifth case will eventually be detected. The delay $\mathrm{d}$ determines how long it takes until this case is eventually diagnosed and interventions are implemented. During this time, the outbreak continues growing exponentially at a rate $r$ determined by the disease-specific parameters $b_{1}, b_{2}, g$ and $\mathrm{k}$. For the Kikwit outbreak this growth rate has been estimated as $\mathrm{r}=0.07 \mathrm{day}^{-1}$, which implies a doubling time of the outbreak of about 10 days [28]. The outbreak size at the time of detection is therefore roughly given by $\mathrm{p}^{-1} \mathrm{e}^{\mathrm{d} \cdot \mathrm{r}}$; for the total outbreak size additional cases from post-intervention period have to be considered. The total number of cases depends on additional parameters such as those describing the effectiveness of the interventions. This means, roughly, that doubling the probability of detection reduces the size of the outbreak by factor 2 as does 
reducing the diagnostic delay by 10 days. Moreover, the effect of reducing the diagnostic delay depends on the growth rate of the outbreak. Reducing diagnostic delays has a stronger impact for faster growing outbreaks.

\section{Discussion}

While VHF outbreaks in Africa have not contributed to disease burdens to the degree seen with malaria, HIV or bacterial infections, they have typically been deadly, remote and unexpected [35]. Information from field epidemiology is critical for preventing and managing outbreaks, and mathematical models can augment information so derived without endangering lives or interfering with patient care. Chowell et al.'s initial SEIR model for Ebola [28] demonstrated that epidemiological interventions such as contact tracing and quarantines halved outbreak size. Legrand et al. [20] recently used a stochastic sixcompartment model to demonstrate that time to intervention is the most important parameter for epidemic control. It may not be cost-effective for resource-limited health systems to test every febrile patient in high-risk areas for Ebola, Marburg, Lassa and other viruses because hemorrhagic viral fever outbreaks are relatively rare and antiviral screening is costly. We therefore employed a model that allowed us to test diagnostic interventions in health workers, a high-risk sub-population and for post-mortems. Recognizing that diagnostic testing is currently not available to most African patients for a myriad of reasons [8,36-38], we focused on diagnostic interventions that could be feasibly introduced to resource-limited hospitals.

We first modeled the impact from enhanced use of inexpensive laboratory tests for common bacterial and parasitic diseases that contribute significantly to the management of those conditions, and reduce selective pressure for resistance to antimalarial and antibacterial drugs. Our working hypothesis was that ruling out these infections in cases of VHF would identify outbreaks early, allowing WHO/CDC-recommended containment protocols to be implemented in a timely manner [6]. By implementing interventions in our modified SEIR model, we demonstrate that an unexpected increase in the number of febrile illness cases not attributable to malaria, typhoid or other endemic diseases, which would be identified by cheap tests, could form the basis of an early warning system that permits outbreak detection sooner than do increases in mortality rates [14]. Thus enhanced laboratory testing for common endemic diseases, such as malaria and bacterial infections, should help to contain uncommon VHF outbreaks. Nationwide rollout of malaria rapid diagnostic testing, which is in progress or strongly advocated for many countries, would have the added benefit of enhancing VHF detection.

Secondly, we tested the hypothesis that thorough diagnostic work-up for febrile health workers, who are at greatest risk of contracting and spreading hemorrhagic viruses in hospitals, would further increase the chance that outbreaks are detected, and therefore contained, early. Investigation of a 1979 Ebola outbreak in rural Sudan revealed that caregivers of Ebola patients had a 5.1-fold increased risk of contracting the disease [39]. In our model, we find that enhanced testing for health workers reduces outbreak size considerably. Focusing on health workers can significantly improve the trajectory of epidemics as well as the safety and morale of those who have the primary responsibility of care and containment $[40,41]$. Protecting health workers also ensures that there will be sufficient personnel to maintain infection control and attend to patients in on-going as well as future epidemics $[15,42]$. This intervention can also bring other benefits, including improved health worker morale, which would be valuable because African health workers have been justifiably reluctant to serve in some hemorrhagic viral outbreaks [16], and increased confidence in the allopathic health care systems as access to them, and their affordability, increases $[15,21,35,43]$.

The third diagnostic testing intervention we evaluated was post-mortem testing. While the results of this intervention compared favorably with the febrile health worker-testing intervention, it may be harder to justify in part because benefits do not accrue to the infected. However it is possible that improved post-mortem testing would prevent burial-associated infections, which are not modeled in this study.

Our study has a number of limitations. It is impossible to replicate all the uncertainty and nuances that accompany an infectious disease epidemic in a theoretical model. Moreover, data from African VHF outbreaks is scanty and of variable quality. The data used in this study were compiled towards the tail end of a devastating outbreak during which record keeping was very likely compromised. These limitations notwithstanding, the model permitted us to test hypotheses for scenarios that would be difficult to test in field studies for logistic as well as ethical reasons. The results demonstrate that although diagnostic tests are not used routinely for most African countries, 
enhanced use, targeted use or both could aid in detecting outbreaks early and minimizing their size.

The majority of patients in Africa with Ebola, Marburg or another VHF receive a clinical case diagnosis only, which is unreliable in the absence of epidemiological cues. WHO-recommended clinical case diagnoses for Ebola and Marburg VHFs require epidemiological connection to a confirmed case $[44,45]$. When laboratory testing is not performed, there are inevitably extended delays to initial outbreak identification. MacNeil et al. [21] have recommended sensitive case surveillance and specific testing, the latter of which will assist in naming epidemics early and ensuring that filovirus-infected patients are segregated from other patients that are critically ill. This VHF contingency strategy requires boosting the epidemiological skills clinical laboratory infrastructure for VHFs in areas where diagnostic support is often sub-par for more common diseases [8,18,21,35]. Remote district facilities are least resourced but most vulnerable because these infections are zoonotic, with reservoirs that are less likely to be encountered in urban environments. A feasible approach to preemptively containing VHF outbreaks would be to implement VHF contingency plans that provide a simultaneous boost to priority areas for health care.

As perhaps best illustrated by the outbreaks in Kikwit (1995), Gulu (2000), Uige (2005) and Bundibugyo (2007), already stretched health systems are invariably overwhelmed by large VHF outbreaks. All those outbreaks saw a loss of confidence in the hospitals that needed to manage and quarantine patients. This suggests that the advantages of early detection may be even greater than those predicted by the model. We did not model outbreaks due to the Lassa arenavirus, because few epidemiologic data are available and a different model, which addresses zoonotic transmission of this agent, would be essential, but the benefits of early diagnosis could be greater for Lassa fever-endemic areas in West Africa. There, Lassa fever infects many more people than all filoviruses combined and accounts for a considerable number of hospitalizations - up to $15 \%$ in highly endemic areas $[46,47]$ - and early treatment with the antiviral ribavarin significantly improves prognosis [48]. As ribavarin is costly, any diagnostic protocol that delineates presumptive Lassa fever cases from malaria will help to target its use. Preventing personto-person transmission of Lassa fever virus, which has been repeatedly documented in health care settings $[7,8,32]$, by early detection could be crucial to preventing additional infections.
The best explanation for the drastic reduction in epidemic size derived from early detection is that the outbreak is contained before or soon after the first round of person-to-person transmission. The risk of person-to-person transmission is very high in African hospitals because febrile patients are not isolated. It is however low once enhanced barrier protocols are instituted following identification of a VHF outbreak. The chance that multiple person-to-person rounds of infection will occur is increased by diagnostic delay. We fully support that improved infection control will have a positive impact on the number and size of VHF epidemics associated with hospitals. Our findings suggest that reducing diagnostic delay through realistic diagnostic protocols that involve enhanced laboratory testing for common endemic diseases, hemorrhagic viruses or both could help determine when more expensive heightened control is required, thereby providing added protection for resource-limited African district hospitals when they need it most.

\section{Conclusions}

African VHFs are among the most lethal but least studied viral infections of humans. We sought to evaluate strategies for containing outbreak size in resource-limited settings and hypothesized that this could be accomplished by routine and judicious use of laboratory diagnostics. Our SEIR model allows us to describe the dynamics for hospital staff separately from that for the general population, and to implement health worker-specific interventions. As a result, we have measured the impact of targeting laboratory diagnostics at highly informative sub-populations. Our results demonstrate that in addition to improving the quality of care for common causes of febrile infections, increased and strategic use of laboratory diagnostics for fever could reduce the chance of hospital amplification of VHFs in resource-limited African health systems.

\section{Acknowledgements}

This work was funded by Branco Weiss Fellowships to INO and TP. We thank Pengfei 'Phil' Zhang and L. Felipe Mosquera for their help with editing and evaluating pilot models for this project.

\section{Author note added to proof}

This paper was submitted in December 2013 and does not incorporate information from the 2014 Ebola outbreak in West Africa. 


\section{References}

1. Berkley JA, Lowe BS, Mwangi I, Williams T, Bauni E, Mwarumba S, Ngetsa C, Slack MP, Njenga S, Hart CA, Maitland K, English M, Marsh K, Scott JA (2005) Bacteremia among children admitted to a rural hospital in Kenya. N Engl J Med 352: 39-47.

2. Gwer S, Newton CR, Berkley JA (2007) Over-diagnosis and co-morbidity of severe malaria in African children: a guide for clinicians. Am J Trop Med Hyg 77: 6-13.

3. Reyburn H, Mbatia R, Drakeley C, Carneiro I, Mwakasungula E, Mwerinde O, Saganda K, Shao J, Kitua A, Olomi R, Greenwood BM, Whitty CJ (2004) Overdiagnosis of malaria in patients with severe febrile illness in Tanzania: a prospective study. Bmj 329: 1212.

4. Evans JA, Adusei A, Timmann C, May J, Mack D, Agbenyega T, Horstmann RD, Frimpong E (2004) High mortality of infant bacteraemia clinically indistinguishable from severe malaria. Qjm 97: 591-597.

5. Peters RP, Zijlstra EE, Schijffelen MJ, Walsh AL, Joaki G, Kumwenda JJ, Kublin JG, Molyneux ME, Lewis DK (2004) A prospective study of bloodstream infections as cause of fever in Malawi: clinical predictors and implications for management. Trop Med Int Health 9: 928-934.

6. WHO, CDC (1998) Infection control for viral haemorrhagic fevers in the African health care setting. 198 p. Available at: http://www.cdc.gov/ncidod/dvrd/spb/pdf/african-healthcaresetting-vhf.pdf Accessed on November 29, 2013

7. Fisher-Hoch SP, Tomori O, Nasidi A, Perez-Oronoz GI, Fakile Y, Hutwagner L, McCormick JB (1995) Review of cases of nosocomial Lassa fever in Nigeria: the high price of poor medical practice. Bmj 311: 857-859.

8. Okeke IN (2011) Divining without seeds : the case for strengthening laboratory medicine in Africa. Ithaca: ILR/ Cornell University Press. 222 p.

9. Amorosa V, MacNeil A, McConnell R, Patel A, Dillon KE, Hamilton K, Erickson BR, Campbell S, Knust B, Cannon D, Miller D, Manning C, Rollin PE, Nichol ST (2010) Imported Lassa fever, Pennsylvania, USA, 2010. Emerg Infect Dis 16: 1598-1600.

10. Bannister B (2010) Viral haemorrhagic fevers imported into non-endemic countries: risk assessment and management. $\mathrm{Br}$ Med Bull 95: 193-225.

11. Atkin S, Anaraki S, Gothard P, Walsh A, Brown D, Gopal R, Hand J, Morgan D (2009) The first case of Lassa fever imported from Mali to the United Kingdom, February 2009. Euro Surveill 14

12. Breman JG, Piot P, Johnson KM, White MK, Mbuyi M, Sureau, P, Heyman, DL, Van Nieuwenhove, S, McCormick, JB, Ruppol, JB, Kintoki, V, Isaacson, M, Van Der Groen, G, Webb, PA, Ngvete, K et al. The epidemiology of ebola haemorrhagic fever in Zaire, 1976. In: Pattyn SR, editor; 1977; Antwerpen, Belgium. Elsevier. pp. 87-99.

13. Fisher-Hoch SP (2005) Lessons from nosocomial viral haemorrhagic fever outbreaks. Br Med Bull 73-74: 123-137.

14. Lamunu M, Lutwama JJ, Kamugisha J, Opio A, Nambooze J, Ndayimirije N, Okware S (2004) Containing a haemorrhagic fever epidemic: the Ebola experience in Uganda (October 2000-January 2001). Int J Infect Dis 8: 27-37.

15. Jeffs B, Roddy $\mathrm{P}$, Weatherill D, de la Rosa O, Dorion C, Iscla M, Grovas I, Palma PP, Villa L, Bernal O, RodriguezMartinez J, Barcelo B, Pou D, Borchert M (2007) The Medecins Sans Frontieres intervention in the Marburg hemorrhagic fever epidemic, Uige, Angola, 2005. I. Lessons learned in the hospital. J Infect Dis 196 Suppl 2: S154-161.

16. Borchert M, Mutyaba I, Van Kerkhove MD, Lutwama J, Luwaga H, Bisoborwa G, Turyagaruka J, Pirard P, Ndayimirije N, Roddy P, Van Der Stuyft P (2011) Ebola haemorrhagic fever outbreak in Masindi District, Uganda: outbreak description and lessons learned. BMC Infect Dis 11: 357.

17. Lamunu M, Lutwama J, Kamugisha J, Opio A, Nambooze J. Containing hemorrhagic fever epidemic, the Ebola experience in Uganda (October 2000-January 2001); 2002; Singapore. World Health Organization. pp. 20pp.

18. Feldmann H, Geisbert TW (2011) Ebola haemorrhagic fever. Lancet 377: 849-862.

19. Adjemian J, Farnon EC, Tschioko F, Wamala JF, Byaruhanga E, Bwire GS, Kansiime E, Kagirita A, Ahimbisibwe S, Katunguka F, Jeffs B, Lutwama JJ, Downing R, Tappero JW, Formenty P, Amman B, Manning C, Towner J, Nichol ST, Rollin PE (2011) Outbreak of Marburg hemorrhagic fever among miners in Kamwenge and Ibanda Districts, Uganda, 2007. J Infect Dis 204 Suppl 3: S796-799.

20. Legrand J, Grais RF, Boelle PY, Valleron AJ, Flahault A (2007) Understanding the dynamics of Ebola epidemics. Epidemiol Infect 135: 610-621.

21. MacNeil A, Farnon EC, Morgan OW, Gould P, Boehmer TK, Blaney DD, Wiersma P, Tappero JW, Nichol ST, Ksiazek TG, Rollin PE (2011) Filovirus outbreak detection and surveillance: lessons from Bundibugyo. J Infect Dis 204 Suppl 3: S761-767.

22. Onyango CO, Opoka ML, Ksiazek TG, Formenty P, Ahmed A, Tukei PM, Sang RC, Ofula VO, Konongoi SL, Coldren RL, Grein T, Legros D, Bell M, De Cock KM, Bellini WJ, Towner JS, Nichol ST, Rollin PE (2007) Laboratory diagnosis of Ebola hemorrhagic fever during an outbreak in Yambio, Sudan, 2004. J Infect Dis 196 Suppl 2: S193-198.

23. Cohen J (2004) Containing the Threat-Don't Forget Ebola. PLoS Med 1: e59.

24. Pittalis S, Fusco FM, Lanini S, Nisii C, Puro V, Lauria FN, Ippolito G (2009) Case definition for Ebola and Marburg haemorrhagic fevers: a complex challenge for epidemiologists and clinicians. New Microbiol 32: 359-367.

25. Bonnet MJ, Akamituna P, Mazaya A (1998) Unrecognized Ebola hemorrhagic fever at Mosango Hospital during the 1995 epidemic in Kikwit, Democratic Republic of the Congo. Emerg Infect Dis 4: 508-510.

26. Groopman JE (2007) How doctors think. Boston: Houghton Mifflin Co.

27. Saijo M, Niikura M, Ikegami T, Kurane I, Kurata T, Morikawa S (2006) Laboratory diagnostic systems for Ebola and Marburg hemorrhagic fevers developed with recombinant proteins. Clin Vaccine Immunol 13: 444-451.

28. Chowell G, Hengartner NW, Castillo-Chavez C, Fenimore PW, Hyman JM (2004) The basic reproductive number of Ebola and the effects of public health measures: the cases of Congo and Uganda. J Theor Biol 229: 119-126.

29. Khan AS, Tshioko FK, Heymann DL, Le Guenno B, Nabeth P, Kerstiëns B, Fleerackers Y, Kilmarx PH, Rodier GR, Nkuku O, Rollin PE, Sanchez A, Zaki SR, Swanepoel R, Tomori O, Nichol ST, Peters CJ, Muyembe-Tamfum JJ, Ksiazek TG (1999) The reemergence of Ebola hemorrhagic fever, Democratic Republic of the Congo, 1995. Commission de Lutte contre les Epidemies a Kikwit. J Infect Dis 179 Suppl 1: S76-86. 
30. Aboderin AO, Adefehinti O, Odetoyin BW, Olotu A, Okeke IN, Adeodu, O. (2012) Prolonged Febrile Illness due to CTXM15 Extended Spectrum b-Lactamase-producing Klebsiella pneumoniae Infection in Nigeria. African Journal of Laboratory Medicine 1: 16.

31. Thiam S, Thior M, Faye B, Ndiop M, Diouf ML, Diallo I, Fall FB, Ndiaye JL, Albertini A, Lee E, Jorgensen P, Gaye O, Bell D (2011) Major reduction in anti-malarial drug consumption in Senegal after nation-wide introduction of malaria rapid diagnostic tests. PLoS ONE 6: e18419.

32. Frame JD, Baldwin JM, Jr., Gocke DJ, Troup JM (1970) Lassa fever, a new virus disease of man from West Africa. I. Clinical description and pathological findings. Am J Trop Med Hyg 19: 670-676.

33. World Health Organization (2003) Outbreak(s) of Ebola haemorrhagic fever in the Republic of the Congo, JanuaryApril 2003. Wkly Epidemiol Rec 78: 285-289.

34. World Health Organization (2005) Outbreak of Ebola haemorrhagic fever in Yambio, south Sudan, April - June 2004. Wkly Epidemiol Rec 80: 370-375.

35. MacNeil A, Rollin PE (2012) Ebola and Marburg Hemorrhagic Fevers: Neglected Tropical Diseases? PLoS Negl Trop Dis 6: e1546.

36. Berkelman R, Cassell G, Specter S, Hamburg M, Klugman K (2006) The "Achilles Heel" of global efforts to combat infectious diseases. Clinical Infectious Diseases 42: 15031504.

37. Petti CA, Polage CR, Quinn TC, Ronald AR, Sande MA (2006) Laboratory medicine in Africa: a barrier to effective health care. Clin Infect Dis 42: 377-382.

38. Polage CR, Bedu-Addo G, Owusu-Ofori A, Frimpong E, Lloyd W, Zurcher E, Hale D, Petti CA (2006) Laboratory use in Ghana: physician perception and practice. Am J Trop Med Hyg 75: 526-531.

39. Baron RC, McCormick JB, Zubeir OA (1983) Ebola virus disease in southern Sudan: hospital dissemination and intrafamilial spread. Bull World Health Organ 61: 997-1003.

40. Hewlett BL, Hewlett BS (2005) Providing care and facing death: nursing during Ebola outbreaks in central Africa. J Transcult Nurs 16: 289-297.

41. Idris A, Idris N (2006) Personal experience: overview of Sudan's experience with Ebola hemorhagic fever (1976). Sudanese Journal of Public Health 1: 49-55.

42. Alsop Z (2007) Ebola outbreak in Uganda "atypical", say experts. Lancet 370: 2085.

43. (2005) Marburg haemorrhagic fever, Angola. Wkly Epidemiol Rec 80: 158-159.

44. WHO (2005) Communicable disease toolkit: Angola. Geneva: World Health Organization.

45. Grolla A, Jones SM, Fernando L, Strong JE, Stroher U, Möller P, Paweska JT, Burt F, Pablo Palma P, Sprecher A, Formenty P, Roth C, Feldmann H (2011) The use of a mobile laboratory unit in support of patient management and epidemiological surveillance during the 2005 Marburg Outbreak in Angola. PLoS Negl Trop Dis 5: e1183.
46. McCormick JB, King IJ, Webb PA, Johnson KM, O'Sullivan R, O'Sullivan R, Smith ES, Trippel S, Tong TC (1987) A case-control study of the clinical diagnosis and course of Lassa fever. J Infect Dis 155: 445-455.

47. Fichet-Calvet E, Rogers DJ (2009) Risk maps of lassa Fever in west Africa. PLoS Negl Trop Dis 3: e388.

48. Richmond JK, Baglole DJ (2003) Lassa fever: epidemiology, clinical features, and social consequences. Bmj 327: 12711275.

49. World Health Organization (2005) Revision of the International Health Regulations. World Health Organization. Fifty-Eighth World Health Assembly A58/4. 60 p.

50. Muyembe-Tamfum JJ, Kipasa M, Kiyungu C, Colebunders R (1999) Ebola outbreak in Kikwit, Democratic Republic of the Congo: discovery and control measures. J Infect Dis 179 Suppl 1: S259-262.

51. Bausch DG, Nichol ST, Muyembe-Tamfum JJ, Borchert M, Rollin PE, Sleurs H, Campbell P, Tshioko FK, Roth C, Colebunders R, Pirard P, Mardel S, Olinda LA, Zeller H, Tshomba A, Kulidri A, Libande ML, Mulangu S, Formenty P, Grein T, Leirs H, Braack L, Ksiazek T, Zaki S, Bowen MD, Smit SB, Leman PA, Burt FJ, Kemp A, Swanepoel R; International Scientific and Technical Committee for Marburg Hemorrhagic Fever Control in the Democratic Republic of the Congo (2006) Marburg hemorrhagic fever associated with multiple genetic lineages of virus. N Engl J Med 355: 909 919.

52. Okware SI, Omaswa FG, Zaramba S, Opio A, Lutwama JJ, Kamugisha J, Rwaguma EB, Kagwa P, Lamunu M (2002) An outbreak of Ebola in Uganda. Trop Med Int Health 7: 10681075.

53. (2003) Outbreak(s) of Ebola haemorrhagic fever, Congo and Gabon, October 2001-July 2002. Wkly Epidemiol Rec 78: 223-228.

54. Leroy EM, Rouquet P, Formenty P, Souquiere S, Kilbourne A, Froment JM, Bermejo M, Smit S, Karesh W, Swanepoel R, Zaki SR, Rollin PE (2004) Multiple Ebola virus transmission events and rapid decline of central African wildlife. Science 303: 387-390.

55. Nkoghe D, Kone ML, Yada A, Leroy E (2011) A limited outbreak of Ebola haemorrhagic fever in Etoumbi, Republic of Congo, 2005. Trans R Soc Trop Med Hyg 105: 466-472.

56. Mason C (2008) The strains of Ebola. Cmaj 178: 1266-1267.

57. Holmes EC (1998) Molecular epidemiology and evolution of emerging infectious diseases. Br Med Bull 54: 533-543.

\section{Corresponding author}

Iruka N Okeke

Department of Biology, Haverford College

370 Lancaster Avenue, Haverford, PA

19041, United States

Phone: +1 6108961470

Email: iokeke@haverford.edu

Conflict of interests: No conflict of interests is declared. 